\title{
Fresnel rhomb and other devices for handling and teaching polarization: inexpensive design
}

\section{B. Zeldovich, I. Ciapurin, H. Sarkissian, C. Tsai}

B. Ya. Zeldovich, I. V. Ciapurin, H. V. Sarkissian, C. Tsai, "Fresnel rhomb and other devices for handling and teaching polarization: inexpensive design," Proc. SPIE 9663, Eighth International Topical Meeting on Education and Training in Optics and Photonics, 966320 (6 October 2003); doi: $10.1117 / 12.2208503$

SPIE Event: Eighth International Topical Meeting on Education and Training in Optics and Photonics, 2003, Tucson, Arizona, United States 


\title{
Fresnel rhomb and other devices for handling and teaching polarization: inexpensive design
}

\author{
B. Ya. Zeldovich, I. V. Ciapurin, H. V. Sarkissian, C. Tsai \\ School of Optics / CREOL, University of Central Florida; 4000 Central Florida Blv, Orlando, FL 32816-2700 \\ Voice (407) 823-6831,FAX (407) 823-6880, boris@creol.ucf.edu
}

\begin{abstract}
We demonstrate a pair of 90-45-45 prisms from binoculars appropriately attached to serve as Fresnel rhomb, i.e. achromatic quarter-wave plate. One and two Dove prisms with metallic reflection instead of TIR can work as half-wave plate and polarization rotator, respectively; both achromatic.

(C)2003 Optical Society of America

OCIS codes: (230.5440) Polarization-sensitive devices, (120.5410) Polarimetry
\end{abstract}

\section{Introduction}

Fresnel rhomb is an important device to study Total Internal Reflection (TIR), circular polarization and other optical phenomena, $[1,2]$. It serves as a quarter-wave plate, which allows transformation of linear polarization into circular or elliptical and back. It uses the fact that in the vicinity of 50 degrees of incidence, one TIR between glass $(n \approx 1.5)$ and air introduces s/p-phase difference about $\pi / 4$, so that two sequential TIRs function as achromatic quarter-wave plate. R. Wood suggests, [3], making Fresnel rhomb by cutting at $54^{\circ}$ two sides of a thick glass plate, then polishing it to a mediocre quality and attaching thin glass plates with Canada balsam to eliminate roughness of polishing. However, the price of Fresnel rhomb is typically 5 to 10 times larger than the price of a right-angle prism.

\section{The devices suggested}

We demonstrate here how a pair of 90-45-45 prisms (right-angle prisms) may be attached to function as Fresnel rhomb, see Figure 1. Immersion liquid was added between the prisms to eliminate parasitic reflections. Small adjustment of the incidence angle, about 5 to 10 degrees, allowed to reach quite good performance of the device as transformer of linear polarization into circular one, with the energy fraction of the "wrong" circular polarization less than 0.0015 at $\lambda=0.6328 \mu \mathrm{m}$.

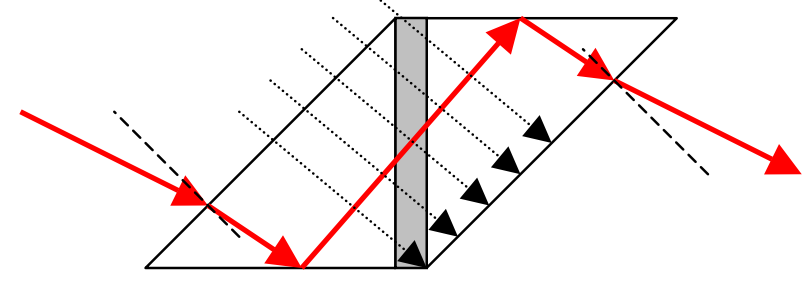

Figure 1. Modified Fresnel rhomb.

4 good right-angle prisms may be extracted from an old and otherwise dysfunctional binocular. It is worth noting that we actually used prisms from a binocular; they had antireflection coatings at the hypotenuse of the prisms and no coatings at short sides. Attempt to introduce light through short sides and use TIRs at hypotenuses yielded 1.41 times wider window, but did not give desired quarter-wave performance due to modification of phases by the coatings. The same device can serve as a demo of a thick flat glass plate (for the rays depicted by dotted arrows).

Dove prisms are conventionally used to rotate image. Most Dove prisms use TIR process for high-quality reflection of light. We suggest here to use metallized reflecting surface, Figure 2. Upper part of the prism is usually cut off. Important property of light reflection from perfect conductor is that the polarization vector is reflected, up to a sign, as an image of a physical object: if $\mathbf{n}$ is the vector of normal to the surface, then

$$
\mathbf{E}_{\text {ref }}=-\mathbf{E}_{\text {inc }}+2 \mathbf{n}\left(\mathbf{n} \cdot \mathbf{E}_{\text {inc }}\right) .
$$

One can see that this prism serves as half-wave plate with respect to polarization, and one of the axes coincides with the normal to the mirror plane. Any prism may be used for that purpose, including (but not limited to) right-angle or equilateral. 


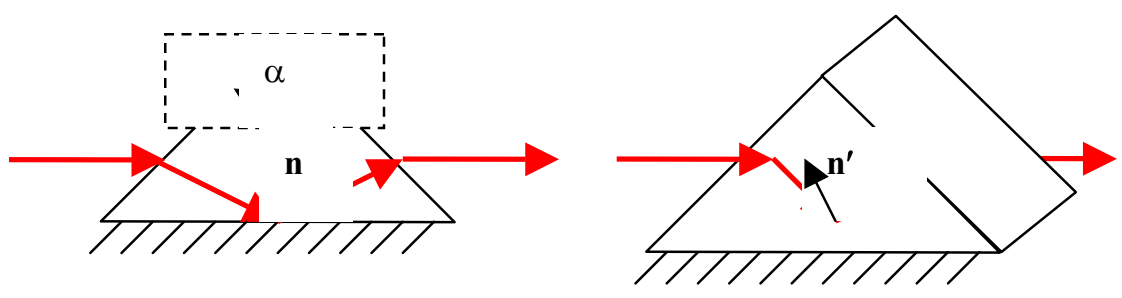

Figure 2. Dove prism modified by metallizing its reflecting surface, and tandem of two such prisms; $\mathbf{n}$ and $\mathbf{n}^{\prime}$ are vectors normal to the mirrors.

Small value of the angle $\alpha$ at the top vortex leads, for a given base length, to relatively small window, but makes less of a change of intensity and polarization at the input and output sides due to Fresnel reflections. A pair (tandem) of two such prisms with an angle $\psi$ between the normal vectors $\mathbf{n}$ and $\mathbf{n}^{\prime}$ to the mirrors yields true rotation of both, the image carried by the beam and the polarization, by the angle $2 \psi$, so that change of $\psi$ allows to continuously rotate the output polarization. It should be emphasized that the action of the above devices in Figure 2 is achromatic, even if the refractive index of the prism's material has arbitrary wavelength dependence. However, high conductivity of the metal and/or highly negative value of its permeability is crucial. While the process of metal deposition is reasonably inexpensive in the conditions of research laboratory or of industry, even simpler solution exists. One may use existing flat mirror, either with front or with back metallized surface, and attach it to the prism with the immersion liquid in-between.

\section{Conclusion}

To conclude, we suggested and demonstrated the performance of 3 inexpensive achromatic devices that function as 1) Fresnel rhomb, 2) thick parallel plate, 3) half-wave plate, and 4) polarization rotator.

\section{References}

1. M. Born, E. Wolf, Principles of Optics, 7-th ed., sect. 1.5.4, Cambridge U. Press, 1999.

2. E Hecht, Optics, 3-rd ed., sect. 8.7, Addison-Wesley, Reading, MA, 1998.

3. R. W. Wood, Physical Optics, 3-rd ed., Chap. IX, p. 354, OSA, Washington DC, 1988. 